\title{
Compton scattering spectroscopy for determining filtration of a clinical computed tomography system
}

\author{
Stella Veloza ${ }^{1}$ \\ Grupo de física nuclear, Departamento de Física, Universidad Nacional de Colombia \\ Av Cra 30 45-03, 111321 Bogotá, Colombia \\ E-mail: Lsvelozas@unal.edu.co

\section{Hans-Ulrich Kauczor} \\ Department of Diagnostic and Interventional Radiology, University Hospital Heidelberg \\ Im Neuenheimer Feld 110, 69120 Heidelberg, Germany \\ E-mail: Hans-Ulrich.Kauczor@med.uni-heidelberg.de
}

\section{Wolfram Stiller}

Department of Diagnostic and Interventional Radiology, University Hospital Heidelberg Im Neuenheimer Feld 110, 69120 Heidelberg, Germany

E-mail: Wolfram.Stilleremed.uni-heidelberg.de

\begin{abstract}
Purpose: Compton spectroscopy is a widely accepted tool for measuring the primary X-ray spectra of a diagnostic computed tomography (CT) system. The aim of this work is to spectroscopically detect CT X-ray photons subjected to 90-degree Compton scattering for accurate modelling of CT beam-shaping filtration, which necessary for computation of radiation exposure and for other research applications in medical physics. Methods: For a tube potential of $120 \mathrm{kV}$ X-ray spectra have been measured under fan angles of $0^{\circ}$ to $20^{\circ}$ in steps of $2^{\circ}$ and at $21^{\circ}$ from the central ray. At each fan angle the value of the X-ray path length determining the relative filter transmission was iteratively varied in order to estimate the thickness of the aluminium filter and the filter geometry was implemented in a Monte Carlo (MC) simulation of the CT scanner. Results: Direct comparison of measured and simulated filtered X-ray spectra indicates that the spectra match very well, with a correlation of 1 for small fan angles (below $4^{\circ}$ ) and an average correlation of 0.996 for the whole field-of-view (FOV). Conclusion: The agreement between the simulated X-ray spectra and the measured spectra suggest that spectral measurements using Compton spectroscopy across the fan beam of a CT system are suitable for modelling the beam-shaping filtration currently used in commercially available CT systems.
\end{abstract}

$X$ Latin American Symposium on Nuclear Physics and Applications (X LASNPA)

1-6 December 2013

Montevideo, Uruguay

${ }^{1}$ Speaker 


\section{Introduction}

Flat X-ray filters of constant thickness and beam-shaping filters are used in CT scanners for removing lower energy X-ray photons (below $20 \mathrm{keV}$ ) which otherwise would be totally absorbed by the patient, increasing patient radiation exposure while not contributing to the image [1]. The thickness of the beam-shaping filter increases from the center to the periphery of the FOV to selectively remove soft X-rays. The characteristics of CT beam-shaping filtration are neither available in user manuals nor in CT technical specifications, because these data are considered proprietary by manufacturers and are available only with vendor cooperation through nondisclosure agreements. Some approaches to determine the attenuation properties of beam-shaping filters have been reported, all based on measurements of the signal after filtration [2-6].

In order to assess the attenuation profile and also the effects of beam-shaping filtration on spectral characteristics, in this research spectral measurements across the fan beam of the CT system have been performed using Compton scattering spectroscopy, an indirect measurement method providing X-ray spectra with the highest accuracy [7-9] however requiring a complex experimental arrangement because clinical X-ray tubes have extremely high photon fluence rates (in the order of $10^{10}$ photons $/ \mathrm{cm}^{2} \mathrm{~s}$ at $100 \mathrm{kV}_{\mathrm{p}}$ and $25 \mathrm{~mA}$ [10]).

\section{Materials and methods}

\subsection{Sequential measurements}

Fig. 1 shows the experimental setup for Compton spectroscopy: the X-ray tube was parked in a stationary position of the CT gantry at three o'clock. A scattering rod within a commercial Compton scattering spectrometer (developed by Matscheko and Ribberfors [8]) mounted on the patient table was initially positioned at the isocenter of the gantry (fan angle $\theta=0^{\circ}$ ). The low energy Germanium detector LEGe (model GL0210, Canberra GmbH, Rüsselsheim, Germany) with an active area of $200 \mathrm{~mm}^{2}$ and a thickness of $10 \mathrm{~mm}$ was placed on the patient table at 22 $\mathrm{cm} \mathrm{z}$-axis distance from the isocenter. The scatterer has a rod geometry $(4 \mathrm{~mm}$ diameter $\times 15$ cm length) and is made of Lucite ${ }^{\mathrm{TM}}\left(\mathrm{C}_{5} \mathrm{H}_{8} \mathrm{O}_{2}\right)_{\mathrm{n}}$ with a density of $1.19 \mathrm{~g} / \mathrm{cm}^{3}$.

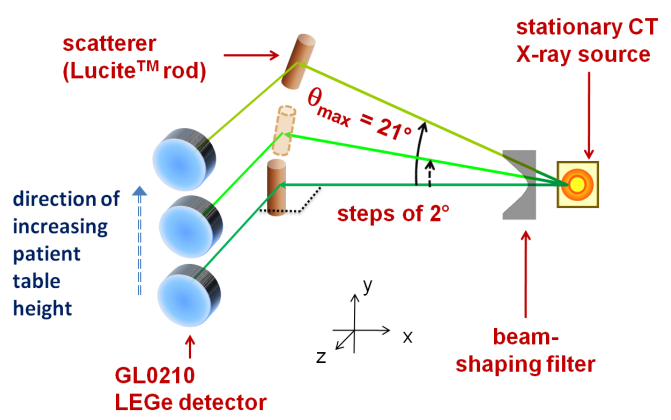

Figure 1: Diagram of the Compton scattering setup for measuring X-ray spectra across the fan beam $\theta$. 


\subsection{Spectral reconstruction}

In order to obtain the beam-shaping filter X-ray spectra as a function of the fan angle, some corrections must be applied to the measured spectra as Compton spectroscopy is an indirect measurement method. These should be made in reverse order from that in which the effects occurred [10], i.e. starting with detection process followed by scattering process. The reconstruction process is explained in the flux diagram of Fig. 2, in which blue boxes symbolize the causes of the spectral distortions, red boxes correspond to the correction steps and orange boxes are the methods and/or data employed for these corrections. After the reconstruction steps a final smoothing of the spectra was performed.

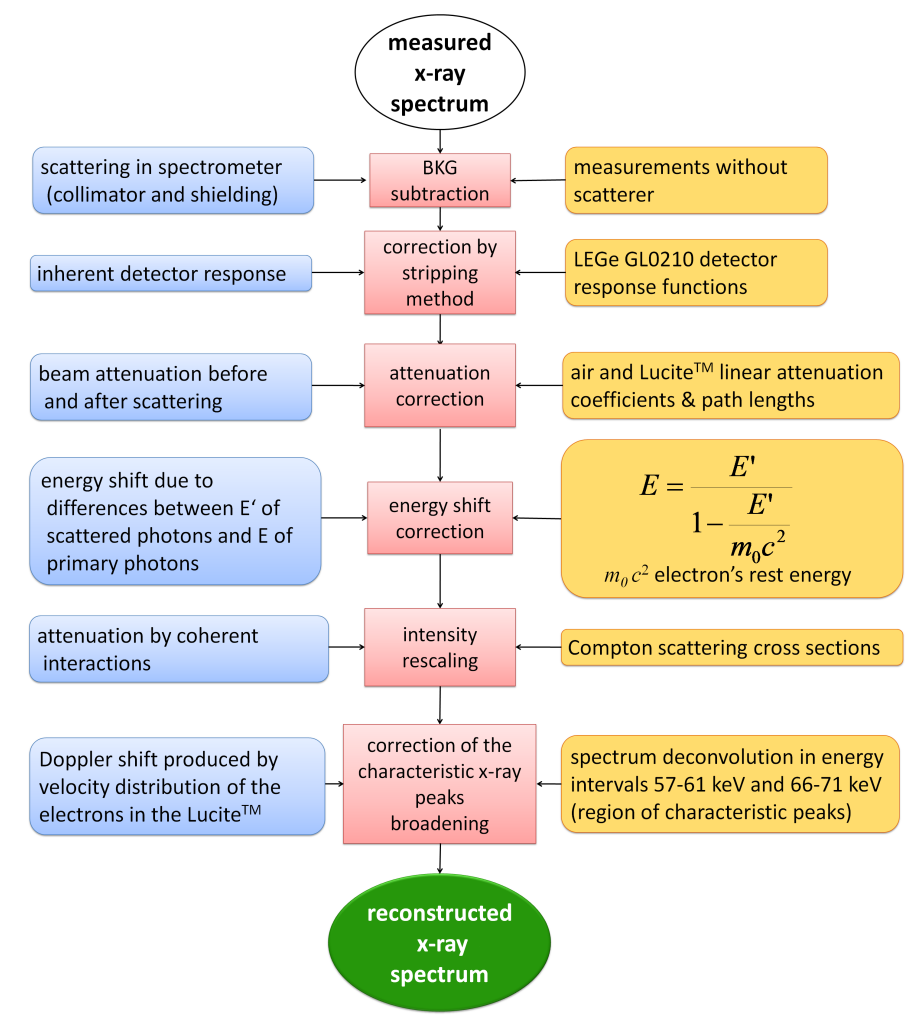

Figure 2: Flux diagram of the spectral correction process for Compton spectroscopy.

\subsection{Beam-shaping filter model}

The hypothesis of the model is that the fan angle-dependent X-ray path length through the beam-shaping filter currently used in $\mathrm{CT}$ systems can be deduced from the attenuation properties of the filter, with some additional assumptions:

1. the filter is symmetric about the center of the X-ray beam at $\theta=0^{\circ}$,

2. the X-ray beam $\Phi_{0}(E)$ incident to the beam-shaping filter includes the effects of the inherent filtration (cooling oil and tube housing window) and any filtration, e.g. flat Xray filtration, made before the beam-shaping filter,

3. The beam-shaping filter is considered being made out of homogeneous aluminium alloy 1100 (Al-1100) material, 
4. only filter transmission $T_{\theta}$ relative to the transmission at the center of the X-ray beam is needed to construct the filter model:

$$
T_{\theta}=\frac{\sum_{i=1}^{n} \Phi_{0}\left(E_{i}\right) e^{-\mu_{A l}\left(E_{i}\right) d_{\theta}} \Delta E}{\sum_{i=1}^{n} \Phi_{0}\left(E_{i}\right) \Delta E},
$$

where $d_{\theta}$ is the relative X-ray path length through the filter (or filter thickness, see Fig. $3)$ at a given fan angle $\theta$ and $\mu_{A l}\left(E_{i}\right)$ are the linear attenuation coefficients of Al-1100 for the $n$ energies $E_{i}$ estimated by interpolation of the National Institute of Standards and Technology (NIST) data [11].

For each fan angle $\theta$ the value of the X-ray path length $d_{\theta}$ is iteratively varied in Eq. 1 until the difference between $T_{\theta}$-computed and the measured transmission $T_{\theta}$-measured is below 0.001 .

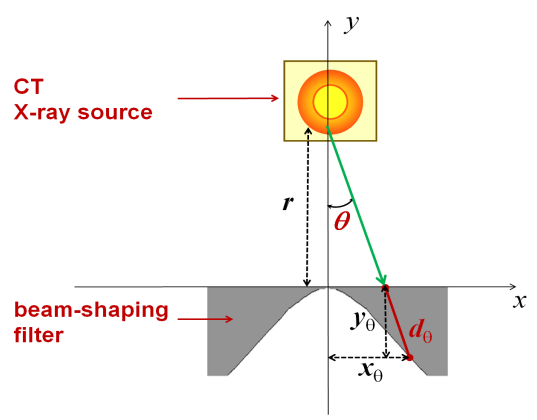

Figure 3: Geometry of the beam-shaping filter model. The filter is symmetric about $\theta=0^{\circ}$. An incident X-ray (green arrow) at a positive fan angle $\theta$ traveling the relative path length $d_{\theta}$ through the filter is illustrated.

\section{Results}

Measured X-ray spectra at various fan angles using the spectral reconstruction procedure described in subsection 2.2 are displayed in Fig. 4(a). The photon fluence of the spectra at large fan angles are strongly reduced in comparison to the photon fluence of the spectra at the center of the X-ray beam (only about $3 \%$ of the photons exiting the filter at $21^{\circ}$ ) and the fraction of low-energy photons (below the peak region around $60 \mathrm{keV}$ ) decreases relative to the fraction of photons with energies greater than the peak region. This effect is caused by beam-hardening.

The relative X-ray path length $d_{\theta}$ through a filter of Al-1100 calculated by iteration was used to construct a MC model of the filter as is illustrated in Fig. 4(b). The filter is bilaterally symmetric and has a uniform thickness along the z-direction $(20.0 \mathrm{~mm})$. The beam's exit points range from $(0.0,0.0) \mathrm{mm}$ at its center to $(76.24,48.60) \mathrm{mm}$ at its edge. 
(a)

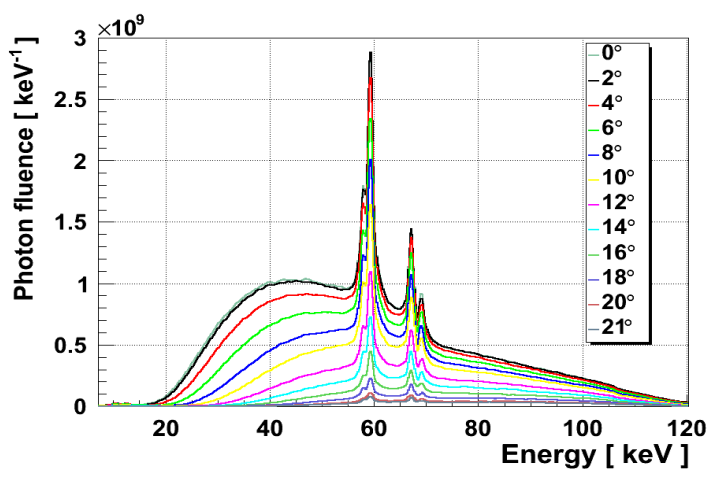

(b)

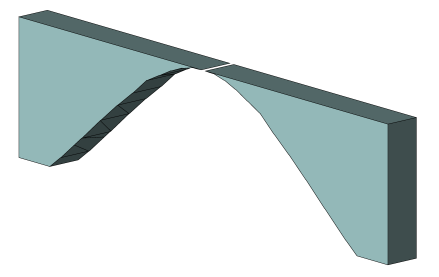

Figure 4: (a) Comparison of X-ray spectra reconstructed from Compton spectrometry measurements at different fan angles. While the overall intensity decreases at larger fan angles, the fraction of high-energy photons increases (beam hardening), (b) 3D rendering of the wedge shape of the beam-shaping filter implemented in the MC simulation.

For direct comparison, the measured and simulated spectra at a given fan angle were normalized to unit area and plotted against each other. Figure 5 exemplary displays the results for a fan angle of $12^{\circ}$. An excellent qualitative agreement can be observed with a correlation of 1 for small fan angles (below $4^{\circ}$ ) and an average correlation of 0.996 for the whole FOV.

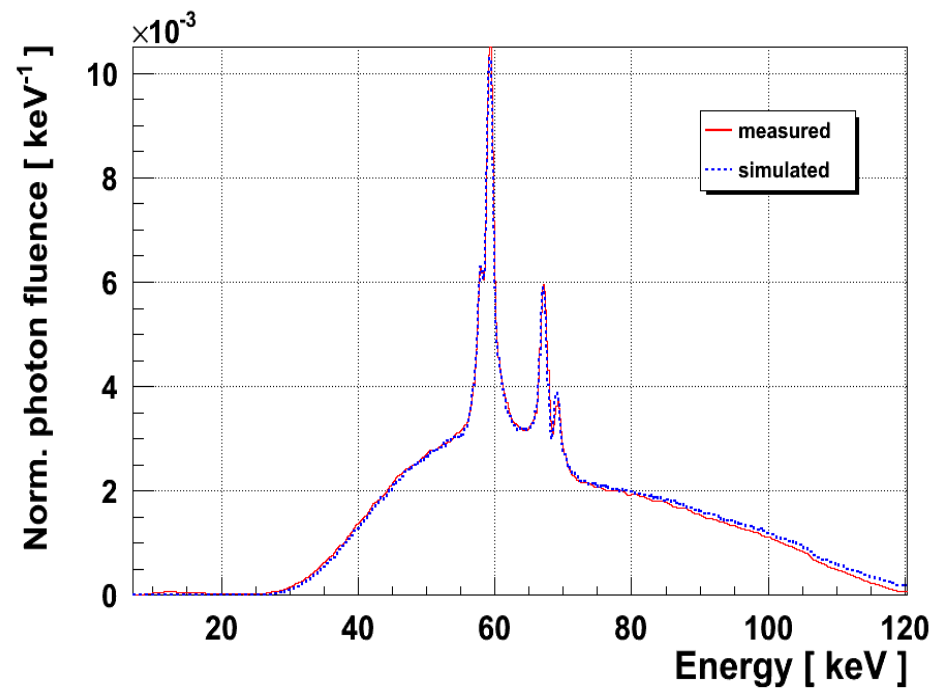

Figure 5: Direct comparison of fan-angle dependent CT X-ray spectra normalized to unit area: spectrum measured by Compton spectrometry (red line) and spectrum simulated (blue line) for a fan angle of $12^{\circ}$.

\section{Conclusions}

Since the characteristics of the beam-shaping filter are vendor proprietary and thus generally unknown, a Compton spectroscopy technique is a suitable tool to model the beamshaping filter with a good agreement between the simulated and measured X-ray spectra. 


\section{References}

[1] M.K. Kalra, M.M. Maher, T.L. Toth, L.M. Hamberg, M.A. Blake, J.A. Shepard and S. Saini, Strategies for CT radiation dose optimization, Radiology 230: 619-628, 2004.

[2] P. Massoumzadeh, S. Don, C.F. Hildebolt, K.T. Bae and B.R. Whiting, Validation of CT dosereduction simulation, Medical Physics 36: 174-189, 2009.

[3] B.R. Whiting, P. Massoumzadeh, O.A. Earl, J.A. O’Sullivan, D.L. Snyder and J.F. Williamson, Properties of preprocessed sinogram data in x-ray computed tomography, Medical Physics 33: 3290-3303, 2006.

[4] A.C. Turner, D. Zhang, H.J. Kim, J.J. DeMarco, C.H. Cagnon, E. Angel, D.D. Cody, D.M. Stevens, A.N. Primak, C.H. McCollough and M. F. McNitt-Gray, A method to generate equivalent energy spectra and filtration models based on measurement for multidetector CT Monte Carlo dosimetry simulations, Medical Physics 36: 2154-2164, 2009.

[5] J. M. Boone, Method for evaluating bow tie filter angle-dependent attenuation in CT: theory and simulation results, Medical Physics 37: 40-48, 2009.

[6] S. E. McKenney, A. Nosratieh, D. Gelskey, K. Yang, S.Y. Huang, L. Chen and J.M. Boone. Experimental validation of a method characterizing bow tie filters in CT scanners using a realtime dose probe, Medical Physics 38: 1406-1415, 2011.

[7] G. Matscheko and R. Ribberfors, A Compton scattering spectrometer for determining X-ray photon energy spectra, Physics in Medicine and Biology 32: 577-594, 1987.

[8] G. Matscheko and G.A. Carlsson, Compton spectroscopy in the diagnostic X-ray energy range: I. Spectrometer design. Physics in Medicine and Biology 34: 185-197, 1989.

[9] G. Matscheko, G.A. Carlsson and R. Ribberfors. Compton spectroscopy in the diagnostic x-ray energy range: II. Effects of scattering material and energy resolution. Physics in Medicine and Biology 34: 199-208, 1989.

[10] M. Yaffe, K.W. Taylor and H.E. Johns, Spectroscopy of diagnostic x rays by a compton-scatter method, Medical Physics 3: 328-334, 1976.

[11] J.H. Hubbel and S.M. Seltzer, Tables of X-ray mass attenuation coefficients and mass energy-absorption coefficients (version 1.4), National Institute of Standards and Technology (NIST). physics.nist.gov/xaamdi. Last access: 23.04.2012, 11:31 